\title{
ON PERTURBATIONS OF AN ODE WITH NON-LIPSCHITZ COEFFICIENTS BY A SMALL SELF-SIMILAR NOISE
}

\author{
ANDREY PILIPENKO AND FRANK NORBERT PROSKE
}

\begin{abstract}
We study the limit behavior of differential equations with non-Lipschitz coefficients that are perturbed by a small self-similar noise. It is proved that the limiting process is equal to the maximal solution or minimal solution with certain probabilities $p_{+}$and $p_{-}=1-p_{+}$, respectively. We propose a space-time transformation that reduces the investigation of the original problem to the study of the exact growth rate of a solution to a certain SDE with self-similar noise. This problem is interesting in itself. Moreover, the probabilities $p_{+}$and $p_{-}$coincide with probabilities that the solution of the transformed equation converges to $+\infty$ or $-\infty$ as $t \rightarrow \infty$, respectively.
\end{abstract}

\section{INTRODUCTION}

We study the limit behavior of the sequence of small-noise stochastic equations

$$
X_{\varepsilon}(t)=\int_{0}^{t}\left(c_{+} \mathbb{1}_{X_{\varepsilon}(s) \geq 0}-c_{-} \mathbb{1}_{X_{\varepsilon}(s)<0}\right)\left|X_{\varepsilon}(s)\right|^{\alpha} d s+\varepsilon B_{\beta}(t),
$$

where $c_{ \pm}>0, \alpha \in(-1,1), B_{\beta}(t), t \geq 0$ is a self-similar process with index $\beta>0$.

Note that the drift $a(x)=\left(c_{+} \mathbb{1}_{x \geq 0}-c_{-} \mathbb{I}_{x<0}\right)|x|^{\alpha} \operatorname{sign} x$ does not satisfy the Lipschitz property at 0 , and the limit equation

$$
X_{0}(t)=\int_{0}^{t} a\left(X_{0}(s)\right) d s, t \geq 0
$$

has two families of solutions

$$
X_{0}^{+, \tau}(t)=\left\{\begin{array}{l}
0, t \in[0, \tau], \\
\left(c_{+}(1-\alpha) t\right)^{\frac{1}{1-\alpha}}, t \geq \tau ;
\end{array} \quad X_{0}^{-, \tau}(t)=\left\{\begin{array}{l}
0, t \in[0, \tau], \\
-\left(c_{-}(1-\alpha) t\right)^{\frac{1}{1-\alpha}}, t \geq \tau,
\end{array}\right.\right.
$$

where $\tau \in[0, \infty]$ is a parameter.

Note that if the self-similar process is a Lévy stable process or a fractional Brownian motion $(\mathrm{fBm})$, then there is a unique solution to the equation (1) under a suitable relation between $\alpha$ and $\beta$, see [1, 2, 3, 9] and Remark 1.2 below. That's why a limit of $\left\{X_{\varepsilon}\right\}$ as $\varepsilon \rightarrow 0$ can be considered as a natural selection for a solution to (2).

The seminal result on a selection problem was a paper of Bafico and Baldi [4] who considered the case where $B_{\beta}$ is a Wiener process. They proved (even for more general form of the drift) that the sequence of distributions of $X_{\varepsilon}$ weakly converges as $\varepsilon \rightarrow 0$ to $p_{-} \delta_{X^{-}}+p_{+} \delta_{X^{+}}$, where $p_{-}+p_{+}=1$ and $X^{ \pm}$are the maximal and the minimal solutions to the limit equation. In our case $X^{ \pm}(t)= \pm\left(c_{ \pm}(1-\alpha) t\right)^{\frac{1}{1-\alpha}}$.

2010 Mathematics Subject Classification. 60H10, 60F99.

Key words and phrases. zero-noise limit; Peano phenomenon; growth rate of solutions to stochastic equations; selection of a solution; self-similar process.

Research is partially supported by FP7-People-2011-IRSES Project number 295164. 
The small-noise problem for non-Lipschitz equations was studied from the different points of view, see [5], [6] and references therein. As for the study of the small-noise problem for SDE's with discontinuous multidimensional drift vector fields we also refer to [7], [8] and [10].

We propose a new approach. We make the following transformation of space and time

$$
\tilde{X}(t):=\tilde{X}_{\varepsilon}(t):=\varepsilon^{\frac{1}{(1-\alpha) \beta-1}} X_{\varepsilon}\left(\varepsilon^{\frac{\alpha-1}{(1-\alpha) \beta-1}} t\right), t \geq 0,
$$

then we use the self similarity property of the process $B_{\beta}$, and show that $\tilde{X}$ satisfies SDE

$$
\tilde{X}(t)=\int_{0}^{t}\left(c_{+} \mathbb{I}_{\widetilde{X}(s) \geq 0}-c_{-} \mathbb{I}_{\widetilde{X}(s)<0}\right)|\widetilde{X}(s)|^{\alpha} d s+\widetilde{B}_{\beta}(t), t \geq 0,
$$

where $\widetilde{B}_{\beta} \stackrel{d}{=} B_{\beta}$. Observe that if (3) has a unique solution, then its distribution is independent of $\varepsilon$. Hence, the limit behavior of $X_{\varepsilon}$ as $\varepsilon \rightarrow 0$ is closely related with the behavior of $\tilde{X}(t)$ as $t \rightarrow \infty$.

Under general conditions a self-similar process with parameter $\beta$ a.s. has a growth that does not exceed $t^{\beta+\delta}$ as $t \rightarrow \infty$ for any $\delta>0$ (see [11, 12, 13]). This holds, for example, if $\tilde{B}$ is a Lévy $\beta^{-1}$-stable process or an $\mathrm{fBm}$. So, if $\alpha+\beta^{-1}>1$, then $\tilde{B}_{\beta}(t)=o\left(\left|X^{ \pm}(t)\right|\right), t \rightarrow \infty$, where $X^{ \pm}= \pm\left(c_{ \pm}(1-\alpha) t\right)^{\frac{1}{1-\alpha}}$. Therefore it is natural to expect that if $\widetilde{X}(t)$ converges to $+\infty$ (or $-\infty$ ) as $t \rightarrow \infty$ and $\alpha+\beta^{-1}>1$, then $\tilde{X}(t) \sim X^{+}(t), t \rightarrow \infty$ (or $\sim X^{-}(t)$ respectively). We prove such result on asymptotic behavior of (small deterministic) perturbation of an integral equation in $\$ 3$. It should be noted that assumption $\alpha+\beta^{-1}>1$ ensures existence of a unique solution if $B_{\beta}$ is a symmetric $\beta^{-1}$-stable Lévy process or $\mathrm{fBm}$, see Remark 1.2. Moreover uniqueness fails if $\alpha+\beta^{-1}<1$, see [2, Theorem 3.2] for a Lévy stable process (the proof of non-uniqueness for fBm is the same).

The application of deterministic results of $\$ 3$ to the study of exact growth rate of solutions to the SDE (3) is given in \$4. This question is interesting by itself. It also may have an application in the construction of consistent estimators for parameters $\alpha$ or $c_{ \pm}$of a solution of equation (3). The first results on exact growth rates of solutions to stochastic differential equations were obtained by Gikhman and Skorokhod [15] who considered SDEs with Wiener noise, see also [16, 17]. We cover some of their results, and our method does not use the Itô formula. Note that cases $\alpha>0$ and $\alpha<0$ are considered separately (here and also in [15]). This is related with monotonicity properties of the function $x^{\alpha}$ and hence with the properties of the limit ODE.

The problem whether $|\tilde{X}(t)| \rightarrow \infty$ as $t \rightarrow \infty$ a.s. is significant, it also appeared in [15]. We prove almost sure convergence to infinity in the case when $B_{\beta}$ is a Lévy stable process and an fBm in \$5, see Examples 1 and 2 .

The small-noise problem for Lévy $\beta$-stable process was solved by Flandoli and Hoegele [18] who even find limit probabilities $p_{ \pm}$. Their method was based on very careful study of jumps of the Lévy process and exits from the neighborhood of 0 .

Our approach allows us to study the small-noise problem even in the case when $X_{\varepsilon}(0)=x_{\varepsilon} \rightarrow$ $0, \varepsilon \rightarrow 0$. Under suitable normalization of $x_{\varepsilon}$, the corresponding limit probabilities $p_{ \pm}$may be different from [18]. Our approach may be useful in the study of the small-noise problem where a drift behaves at 0 "similarly" to $\left(c_{+} \mathbb{I}_{x \geq 0}-c_{-} \mathbb{I}_{x<0}\right)|x|^{\alpha}$, or in the case when the noise a Lévy process whose Lévy measure in a neighborhood of zero is "close" to Lévy measure of Lévy $\beta$ stable process. We also plan to study the multiplicative noise. 


\section{Main Result}

Let $B_{\beta}(t), t \geq 0$, be a self-similar process with index $\beta>0$, i.e., for any $a>0$ the distribution of processes $\left\{B_{\beta}(a t), t \geq 0\right\}$ and $\left\{a^{\beta} B_{\beta}(t), t \geq 0\right\}$ are equal. We will always assume that all considered processes have cádlág trajectories.

Consider stochastic equation

$$
X_{\varepsilon}(t)=\int_{0}^{t}\left(c_{+} \mathbb{I}_{X_{\varepsilon}(s) \geq 0}-c_{-} \mathbb{I}_{X_{\varepsilon}(s)<0}\right)\left|X_{\varepsilon}(s)\right|^{\alpha} d s+\varepsilon B_{\beta}(t), t \geq 0,
$$

where $c_{ \pm}>0$.

By a weak solution to (4) we call a pair of adapted processes $\left(\tilde{X}, \tilde{B}_{\beta}\right)$ defined on a filtered probability space $\left(\Omega, \mathcal{F}, P,\left\{\mathcal{F}_{t}, t \geq 0\right\}\right)$ such that

1) $\left(\tilde{X}, \tilde{B}_{\varepsilon}\right)$ satisfy (4) a.s.,

2) $\tilde{B}_{\beta} \stackrel{d}{=} B_{\beta}$.

Further we will omit tilde in the notation and assume that all processes are defined on the corresponding probability space.

If $B_{\beta}$ is a Lévy process, then we naturally assume that $\tilde{B}_{\beta}$ is $\mathcal{F}_{t}$-Lévy process. If $B_{\beta}$ is an $\mathrm{fBm}$, we make the same assumption on $\mathcal{F}_{t}$ as in [3].

The weak uniqueness means that all weak solutions have the same distribution.

Theorem 1.1. Let $\alpha+\beta^{-1}>1$. Assume that

(1) there exists a unique weak solution to the equation

$$
X(t)=\int_{0}^{t}\left(c_{+} \mathbb{I}_{X(s) \geq 0}-c_{-} \mathbb{I}_{X(s)<0}\right)|X(s)|^{\alpha} d s+B_{\beta}(t), t \geq 0
$$

(2) $\mathrm{P}\left(\lim _{t \rightarrow \infty}|X(t)|=\infty\right)=1$;

(3) $\alpha \in(0,1)$ and there exists a function $h(t)=o\left(t^{\frac{1}{1-\alpha}}\right), t \rightarrow \infty$, and a random sequence $\left\{t_{n}\right\}, \lim _{n \rightarrow \infty} t_{n}=\infty$, such that $\left|B_{\beta}(t)-B_{\beta}\left(t_{n}\right)\right| \leq h\left(t-t_{n}\right), t \geq t_{n}$, a.s.

or

$\alpha \in(-1,0]$ and $B_{\beta}(t)=o\left(t^{\frac{1}{1-\alpha}}\right), t \rightarrow \infty$ a.s.

Then the sequence of distributions of processes $\left\{X_{\varepsilon}\right\}$ converges in $D([0, \infty))$ to the distribution of the process

$$
((1-\alpha) t)^{\frac{1}{1-\alpha}}\left(c_{+}^{\frac{1}{1-\alpha}} \mathbb{I}_{\left\{\lim _{t \rightarrow \infty} X(t)=+\infty\right\}}-c_{-}^{\frac{1}{1-\alpha}} \mathbb{I}_{\left\{\lim _{t \rightarrow \infty} X(t)=-\infty\right\}}\right) .
$$

Remark 1.1. The limit process equals $X^{+}(t)=\left(c_{+}(1-\alpha) t\right)^{\frac{1}{1-\alpha}}$ or $X^{-}(t)=-\left(c_{-}(1-\alpha) t\right)^{\frac{1}{1-\alpha}}$ with probabilities

$$
p_{ \pm}=\mathrm{P}\left(\lim _{t \rightarrow \infty} X(t)= \pm \infty\right)
$$

respectively. Functions $X^{ \pm}(t), t \geq 0$, are the maximal positive solution to the ODE

$$
y^{\prime}(t)=c_{+} y^{\alpha}(t), t \geq 0, y(0)=0,
$$

and the minimal negative solution to the ODE

$$
y^{\prime}(t)=-c_{-}|y|^{\alpha}(t), t \geq 0, y(0)=0,
$$

respectively. 
Remark 1.2. Assume that $B_{\beta}$ is a symmetric $\beta^{-1}$ Lévy stable process and $\alpha+\beta^{-1}>1$. Then equation (5) has a unique (strong) solution. The case $\alpha>0$ was proved in [2]. The existence of a weak solution for $\alpha \leq 0$ follows from [9], uniqueness was proved in [14].

If $B_{\beta}$ is a Brownian motion, then $\beta=1 / 2$, and, see for example [1], equation (5) has a unique solution if $\alpha>-1$, i.e., $\alpha+(1 / 2)^{-1}>1$.

If $B_{\beta}$ is a fractional Brownian motion with Hurst parameter $H=\beta$, then (strong) existence and uniqueness holds for $H \in(0,1)$ and $\alpha \geq 0$, see [3]. If $H \in(1,2)$, it was proved in [3] that (strong) existence uniqueness holds for $\alpha+0.5 H^{-1}>1$. However their method works for $\alpha+H^{-1}>1$. Indeed, it follows from their estimates that (see [3, page 110] with their notations)

$$
\begin{gathered}
\forall \varepsilon>0 \exists K_{1}, K_{2}>0 \quad \int_{0}^{T} \beta^{2}(s) d s \leq \cdots \leq K_{1} \int_{0}^{T} s^{1-2 H+2 \alpha(H-\varepsilon)} d s G^{2 \alpha}= \\
K_{2} G^{2 \alpha}<\infty
\end{gathered}
$$

if $1-2 H+2 \alpha(H-\varepsilon)>-1$, that is, $\alpha+(H-\varepsilon)^{-1}>\frac{H}{H-\varepsilon}$. The bound $\int_{0}^{T} \beta^{2}(s) d s \leq K_{2} G^{2 \alpha}$ was sufficient for existence and uniqueness. Since $\varepsilon>0$ was arbitrary, we have existence and uniqueness if $\alpha+H^{-1}>1$.

Remark 1.3. Condition 3 of the Theorem is satisfied for a Lévy stable process or a fractional Brownian motion. Indeed, it follows from [11, 12] that for any $\varepsilon>0$

$$
\lim _{t \rightarrow \infty} \frac{B_{\beta}(t)}{t^{\beta+\varepsilon}}=0 \text { a.s. }
$$

Let $M>0$ and $\varepsilon \in\left(0, \frac{1}{1-\alpha}-\beta\right)$. Set

$$
\begin{gathered}
h_{M, \varepsilon}(t):=M+t^{\beta+\varepsilon}=o\left(t^{\frac{1}{1-\alpha}}\right), t \rightarrow \infty, \\
A_{M, \varepsilon}=\left\{\forall t \geq 0:\left|B_{\beta}(t)\right| \leq h_{M, \varepsilon}(t)\right\} .
\end{gathered}
$$

It follows from the Poincaré recurrence theorem that

$$
\mathrm{P}\left(A_{M, \varepsilon} \cap\left\{\exists N \forall n \geq N \exists t \geq 0:\left|B_{\beta}(t+n)-B_{\beta}(n)\right| \geq h_{M, \varepsilon}(t)\right\}\right)=0 .
$$

Since $\lim _{M \rightarrow+\infty} \mathrm{P}\left(A_{M, \varepsilon}\right)=1$, (6) implies condition 3 of the Theorem.

Verification of condition 2 for these noises is given in $\$ 4$.

\section{TRANSFORMATIONS OF THE MAIN EQUATION}

Let $B_{\beta}(t), t \geq 0$ be a self-similar process with index $\beta>0$.

By $X_{x, c, \alpha, \varepsilon}(t)$ denote a (weak) solution of

$$
X(t)=x+\int_{0}^{t}\left(c_{+} \mathbb{\Pi}_{X(s) \geq 0}-c_{-} \mathbb{I}_{X(s)<0}\right)|X(s)|^{\alpha} d s+\varepsilon B_{\beta}(t) .
$$

Lemma 2.1. Let Xbe a solution of (7). Then the process $\tilde{X}(t):=\varepsilon^{\delta} X\left(\varepsilon^{-\gamma} t\right)$ is a solution of

$$
\begin{gathered}
\widetilde{X}(t)=x \varepsilon^{\delta}+\varepsilon^{\delta(1-\alpha)-\gamma} \int_{0}^{t}\left(c_{+} \mathbb{I}_{\widetilde{X}(s) \geq 0}-c_{-} \mathbb{I}_{\widetilde{X}(s)<0}\right)|\widetilde{X}(s)|^{\alpha} d s+ \\
\varepsilon^{1+\delta-\gamma \beta} \widetilde{B}_{\beta}(t), t \geq 0,
\end{gathered}
$$

where $\widetilde{B}_{\beta} \stackrel{d}{=} B_{\beta}$. 
ON PERTURBATIONS OF AN ODE WITH NON-LIPSCHITZ COEFFICIENTS BY A SMALL SELF-SIMILAR NOISE 5

That is, the distributions of

$$
\varepsilon^{\delta} X_{x, c, \alpha, \varepsilon}\left(\varepsilon^{-\gamma} t\right), t \geq 0, \text { and } X_{x \varepsilon^{\delta}, c \varepsilon^{\delta(1-\alpha)-\gamma}, \alpha, \varepsilon^{1+\delta-\gamma \beta}}(t), t \geq 0,
$$

are equal if at least one of equations (7) or (8) has a unique weak solution.

In particular, if $x=0, \delta=\frac{1}{(1-\alpha) \beta-1}, \gamma=\frac{1-\alpha}{(1-\alpha) \beta-1}$, then $\widetilde{X}(t)$ satisfies the equation (3).

Proof.

$$
\begin{gathered}
\tilde{X}(t)=\varepsilon^{\delta} X\left(\varepsilon^{-\gamma} t\right)=x \varepsilon^{\delta}+\varepsilon^{\delta} \int_{0}^{\varepsilon^{-\gamma} t}\left(c_{+} \mathbb{I}_{X(s) \geq 0}-c_{-} \mathbb{I}_{X(s)<0}\right)|X(s)|^{\alpha} d s+ \\
\varepsilon^{\delta} \varepsilon B_{\beta}\left(\varepsilon^{-\gamma} t\right)= \\
x \varepsilon^{\delta}+\varepsilon^{\delta} \int_{0}^{t}\left(c_{+} \mathbb{I}_{X\left(z \varepsilon^{-\gamma}\right) \geq 0}-c_{-} \mathbb{I}_{X\left(z \varepsilon^{-\gamma}\right)<0}\right)\left|X\left(z \varepsilon^{-\gamma}\right)\right|^{\alpha} d z \varepsilon^{-\gamma}+ \\
+\varepsilon^{\delta} \varepsilon \varepsilon^{-\gamma \beta} \varepsilon^{\gamma \beta} B_{\beta}\left(\varepsilon^{-\gamma} t\right)= \\
x \varepsilon^{\delta}+\varepsilon^{\delta} \varepsilon^{-\gamma} \varepsilon^{-\delta \alpha} \int_{0}^{t}\left(c_{+} \mathbb{I}_{\widetilde{X}(z) \geq 0}-c_{-} \mathbb{I}_{\widetilde{X}(z)<0}\right)|\widetilde{X}(z)|^{\alpha} d z+ \\
+\varepsilon^{1+\delta-\gamma \beta} \widetilde{B}_{\beta}(t),
\end{gathered}
$$

where $\widetilde{B}_{\beta}(t)=\varepsilon^{\gamma \beta} B_{\beta}\left(\varepsilon^{-\gamma} t\right)$.

Corollary 2.1. Let $X$ be a solution of (5), $X_{\varepsilon}$ be a solution of (4). Then

$$
\left\{X_{\varepsilon}(t), t \geq 0\right\} \stackrel{d}{=}\left\{\varepsilon^{\frac{-1}{(1-\alpha) \beta-1}} X\left(\varepsilon^{\frac{1-\alpha}{(1-\alpha) \beta-1}} t\right), t \geq 0\right\}
$$

if at least one of equations (5) or (4) has a unique weak solution.

\section{ASYMPTOTIC BEHAVIOR OF PERTURBED INTEGRAL EQUATION}

Let $c>0, x>0$. In this section we find sufficient conditions ensuring that a solution to the integral equation

$$
y(t)=x+c \int_{0}^{t}|y(s)|^{\alpha} d s+g(t)
$$

is equivalent as $t \rightarrow \infty$ to the solution of

$$
z(t)=x+c \int_{0}^{t}|z(s)|^{\alpha} d s
$$

if the function $g$ has comparatively small growth on infinity. I.e., we will find conditions on growth of $g$ that suffices

$$
y(t) \sim(c(1-\alpha) t)^{\frac{1}{1-\alpha}}, t \rightarrow \infty .
$$

Let $X$ be a solution of (5). Under natural integrability assumptions [11, 12, 13], self-similar process $B_{\beta}$ satisfies the following growth condition

$$
\forall \delta>0 \quad \lim _{t \rightarrow \infty} \frac{B_{\beta}(t)}{t^{\beta+\delta}}=0 \text { a.s. }
$$

So, it is natural to expect that if $\omega \in \Omega$ is such that $X(t)=X(t, \omega) \rightarrow+\infty, t \rightarrow \infty$, then $X(t, \omega) \sim\left(c_{+}(1-\alpha) t\right)^{\frac{1}{1-\alpha}}, t \rightarrow \infty\left(\right.$ or $X(t) \sim-\left(c_{-}(1-\alpha) t\right)^{\frac{1}{1-\alpha}}$ if $\lim _{t \rightarrow \infty} X(t)=-\infty$, respectively). We apply deterministic result on equivalence (10) to the stochastic equation (4) in the next section. 
We consider the cases $\alpha \in(0,1)$ and $\alpha \in(-1,0]$ separately. In these cases the function $x^{\alpha}$ is increasing or decreasing, respectively. This has an effect on properties of the solution and the course of the proof.

We will always assume that all functions below are measurable and locally bounded.

3.1. Case $\alpha \in(0,1)$. In order to study the asymptotic behavior of $y(t)$ we need few simple auxiliary lemmas.

Lemma 3.1. Let $a: \mathbb{R} \rightarrow \mathbb{R}$ be a non-decreasing, locally Lipschitz function of linear growth, $g_{1}, g_{2}:[0, T] \rightarrow \mathbb{R}$ be bounded measurable functions,

$$
y_{i}(t)=x_{i}+\int_{0}^{t} a\left(y_{i}(s)\right) d s+g_{i}(t), t \in[0, T], i=1,2 .
$$

Assume that $x_{1} \leq x_{2}, g_{1}(t) \leq g_{2}(t), t \in[0, T]$. Then $y_{1}(t) \leq y_{2}(t), t \in[0, T]$.

Assumptions of the lemma yield that the solutions are unique and can be obtained by iterations. The corresponding inequality obviously is satisfied for iterations, so it is satisfied for their limits too.

Remark 3.1. The assumption on monotonicity of $a$ cannot be omitted.

As a corollary of Lemma 3.1 we get the following result.

Lemma 3.2. Let $y_{1}(t) \geq \varepsilon>0, t \in[0, T]$. Assume that $y_{1}(t), y_{2}(t), t \in[0, T]$ are such that

$$
y_{1}(t) \leq x_{1}+c \int_{0}^{t} y_{1}^{\alpha}(s) d s+g_{1}(t), t \in[0, T]
$$

and

$$
y_{2}(t) \geq x_{2}+c \int_{0}^{t} y_{2}^{\alpha}(s) d s+g_{2}(t), t \in[0, T], i=1,2,
$$

where $\alpha \in(0,1), g_{1}, g_{2}:[0, T] \rightarrow \mathbb{R}$ are bounded measurable functions.

If $x_{1} \leq x_{2}, g_{1}(t) \leq g_{2}(t), t \in[0, T]$, then $y_{1}(t) \leq y_{2}(t), t \in[0, T]$.

Theorem 3.1. Assume that $y(t)$ satisfies the equation

$$
y(t)=x+c \int_{0}^{t}|y(s)|^{\alpha} d s+g(t), t \geq 0,
$$

where $c>0, \alpha \in(0,1), g:[0, \infty) \rightarrow \mathbb{R}$ is a measurable locally bounded function. Assume that $\lim _{t \rightarrow \infty} y(t)=+\infty$ and there exists a sequence $\left\{t_{n}\right\}, \lim _{n \rightarrow \infty} t_{n}=\infty$ and a function $h(t)=$ $o\left(t^{\frac{1}{1-\alpha}}\right), t \rightarrow \infty$, such that

$$
\left|g(t)-g\left(t_{n}\right)\right| \leq h\left(t-t_{n}\right), t \geq t_{n}
$$

Then

$$
y(t) \sim(c(1-\alpha) t)^{\frac{1}{1-\alpha}}, t \rightarrow \infty .
$$

Remark 3.2. Assumption $\lim _{t \rightarrow \infty} y(t)=+\infty$ cannot be omitted. Indeed, $y(t)=0, t \geq 0$, satisfies the equation with $x=0, g \equiv 0$. The condition $\left|g(t)-g\left(t_{n}\right)\right| \leq h\left(t-t_{n}\right), t \geq t_{n}$ also cannot be replaced by $g(t)=o\left(t^{\frac{1}{1-\alpha}}\right), t \rightarrow \infty$. Indeed, let $\gamma \in\left(0, \frac{1}{1-\alpha}\right), y(t)=t^{\gamma}, t_{0}=0, x=0$. Then

$$
y(t)=\int_{0}^{t}\left(s^{\gamma}\right)^{\alpha} d s-\int_{0}^{t}\left(s^{\gamma}\right)^{\alpha} d s+t^{\gamma}=
$$


ON PERTURBATIONS OF AN ODE WITH NON-LIPSCHITZ COEFFICIENTS BY A SMALL SELF-SIMILAR NOISE 7

$$
=\int_{0}^{t}|y(s)|^{\alpha} d s-\frac{t^{\alpha \gamma+1}}{\alpha \gamma+1}+t^{\gamma}=\int_{0}^{t}|y(s)|^{\alpha} d s+g(t),
$$

where $g(t)=-\frac{t^{\alpha \gamma+1}}{\alpha \gamma+1}+t^{\gamma}$.

Since $\alpha \gamma+1<\frac{\alpha}{1-\alpha}+1=\frac{1}{1-\alpha}$, we have $g(t)=o\left(t^{\frac{1}{1-\alpha}}\right)$ and $f(t)=o\left(t^{\frac{1}{1-\alpha}}\right)$ as $t \rightarrow \infty$.

Proof of Theorem 3.1. We have

$$
y(t)=y\left(t_{n}\right)+c \int_{t_{n}}^{t}|y(s)|^{\alpha} d s+g(t)-g\left(t_{n}\right) \geq y\left(t_{n}\right)+c \int_{t_{n}}^{t}|y(s)|^{\alpha} d s+h\left(t-t_{n}\right), t \geq t_{n} .
$$

Set $z_{n}(t)=y\left(t+t_{n}\right), x_{n}=y\left(t_{n}\right)$. Then

$$
z_{n}(t) \geq x_{n}+c \int_{0}^{t}\left|z_{n}(s)\right|^{\alpha} d s-h(t), t \geq 0
$$

For $a>0, t \geq 0$ denote $\widetilde{z}^{(a)}(t)=(1+a(1-\alpha) t)^{\frac{1}{1-\alpha}}$. The function $\widetilde{z}^{(a)}$ satisfies the equation

$$
\widetilde{z}^{(a)}(t)=1+a \int_{0}^{t} \widetilde{z}^{(a)}(s)^{\alpha} d s .
$$

Let $c_{-}<c$. Then

$$
\begin{gathered}
\widetilde{z}^{\left(c_{-}\right)}(t)=1+c_{-} \int_{0}^{t} \widetilde{z}^{\left(c_{-}\right)}(s)^{\alpha} d s= \\
1+c \int_{0}^{t} \widetilde{z}^{\left(c_{-}\right)}(s)^{\alpha} d s+\left(c_{-}-c\right) \int_{0}^{t} \widetilde{z}^{\left(c_{-}\right)}(s)^{\alpha} d s= \\
=1+c \int_{0}^{t} \widetilde{z}^{\left(c_{-}\right)}(s)^{\alpha} d s+\left(c_{-}-c\right)\left(\left(1+c_{-}(1-\alpha) t\right)^{\frac{1}{1-\alpha}}-1\right) \leq \\
\leq K\left(c_{-}\right)+c \int_{0}^{t} \widetilde{z}^{\left(c_{-}\right)}(s)^{\alpha} d s-h(t), t \geq 0,
\end{gathered}
$$

where $K\left(c_{-}\right)$is a constant.

It follows from (12), (13), and Lemma 3.2 that if $n$ is sufficiently large, then $y\left(t_{n}+t\right)=z_{n}(t) \geq$ $\widetilde{z}^{\left(c_{-}\right)}(t), t \geq 0$. So

$$
\varlimsup_{t \rightarrow \infty} \frac{y_{n}(t)}{\left(1+c_{-}(1-\alpha) t\right)^{\frac{1}{1-\alpha}}} \geq 1
$$

Therefore

$$
\forall c_{-}<c \quad \varlimsup_{t \rightarrow \infty} \frac{y(t)}{(c(1-\alpha) t)^{\frac{1}{1-\alpha}}} \geq \frac{c_{-}^{\frac{1}{1-\alpha}}}{c^{\frac{1}{1-\alpha}}} .
$$

Similarly we get the inequality

$$
\forall c_{+}>c \quad \underline{\lim }_{t \rightarrow \infty} \frac{y(t)}{(c(1-\alpha) t)^{\frac{1}{1-\alpha}}} \leq \frac{c_{+}^{\frac{1}{1-\alpha}}}{c^{\frac{1}{1-\alpha}}} .
$$

Since $c_{-}<c$ and $c_{+}>c$ were arbitrary, this proves the theorem. 
3.2. Case $\alpha \in(-1,0]$. In this subsection we assume that solution of (9) is positive for all $t \geq 0$.

Lemma 3.3. Let $\varepsilon \in(0,1), a>1, \Delta \in\left(0, \frac{1+\alpha}{-\alpha}\right) \cap\left(0, \frac{1}{\varepsilon}-1\right)$ be arbitrary. There exists $\delta=$ $\delta(\varepsilon, a, \Delta)>0$ depending only on $\varepsilon, a, \Delta$ such that if

$$
|g(t)| \leq \delta t^{\frac{1}{1-\alpha}}, t \in\left[a^{n}, a^{n+1}\right],
$$

for some $n \geq 1$ and $y$ is a solution of (9) such that

$$
(1-\varepsilon)\left(c(1-\alpha) a^{n}\right)^{\frac{1}{1-\alpha}}<y\left(a^{n}\right)<(1+\varepsilon)\left(c(1-\alpha) a^{n}\right)^{\frac{1}{1-\alpha}},
$$

then for all $t \in\left[a^{n}, a^{n+1}\right]$ we have

$$
(1-\varepsilon(1+\Delta))\left(c(1-\alpha) a^{n}\right)^{\frac{1}{1-\alpha}}<y(t)<(1+\varepsilon)\left(c(1-\alpha) a^{n+1}\right)^{\frac{1}{1-\alpha}},
$$

and

$$
(1-\varepsilon)\left(c(1-\alpha) a^{n+1}\right)^{\frac{1}{1-\alpha}}<y\left(a^{n+1}\right) .
$$

Proof. The function $x^{\alpha}, x>0$ is decreasing. So, if

$$
0<y_{-}(t)<y(t)<y_{+}(t), t \in\left[a^{n}, a^{n+1}\right]
$$

then for all $t \in\left[a^{n}, a^{n+1}\right]$

$$
\begin{gathered}
y(t)=y\left(a^{n}\right)+\int_{a^{n}}^{t} c y^{\alpha}(s) d s+g(t)-g\left(a^{n}\right)< \\
y_{+}\left(a^{n}\right)+\int_{a^{n}}^{t} c y_{-}^{\alpha}(s) d s+|g(t)|+\left|g\left(a^{n}\right)\right| \leq \\
y_{+}\left(a^{n}\right)+c \max _{s \in\left[a^{n}, t\right]} y_{-}^{\alpha}(s)\left(t-a^{n}\right)+2 \delta t^{\frac{1}{1-\alpha}} \leq \\
y_{+}\left(a^{n}\right)+c a^{n}(a-1) \max _{s \in\left[a^{n}, a^{n+1}\right]} y_{-}^{\alpha}(s)+2 \delta a^{\frac{n+1}{1-\alpha}} .
\end{gathered}
$$

Similarly

$$
y(t)>y_{-}\left(a^{n}\right)+c a^{n}(a-1) \min _{s \in\left[a^{n}, a^{n+1}\right]} y_{+}^{\alpha}(s)-2 \delta a^{\frac{n+1}{1-\alpha}} .
$$

By $y_{-}$and $y_{+}$denote the left hand side and the right hand side of (15), correspondingly. To prove the lemma it suffices to show that there exists $\delta>0$ such that the upper (and the lower) bound of $y$ from inequalities (17) (respectively (18)) is less than the right hand side of (15) (is greater than the left hand side of (15) and (16)).

Let us check the upper bound only. The lower bound can be proved similarly.

Let $t \in\left[a^{n}, a^{n+1}\right]$. We have to verify

$$
\begin{gathered}
(1+\varepsilon)\left(c(1-\alpha) a^{n}\right)^{\frac{1}{1-\alpha}}+c a^{n}(a-1)\left((1-\varepsilon(1+\Delta))\left(c(1-\alpha) a^{n}\right)^{\frac{1}{1-\alpha}}\right)^{\alpha}+2 \delta a^{\frac{n+1}{1-\alpha}}< \\
(1+\varepsilon)\left(c(1-\alpha) a^{n+1}\right)^{\frac{1}{1-\alpha}}
\end{gathered}
$$

or the following equivalent inequality

$$
\frac{(1-\varepsilon(1+\Delta))^{\alpha}(a-1)}{1-\alpha}+\frac{2 \delta a^{\frac{1}{1-\alpha}}}{(c(1-\alpha))^{\frac{1}{1-\alpha}}}<(1+\varepsilon)\left(a^{\frac{1}{1-\alpha}}-1\right)
$$

for some fixed $\delta=\delta(\varepsilon, a, \Delta)>0$. 
Since $\alpha \in(-1,0]$, the mean value theorem yields inequalities

$$
\forall x \in(0,1)(1-x)^{\alpha} \leq 1-\alpha x ; \quad \forall x>0 x^{\frac{1}{1-\alpha}}-1 \geq \frac{x-1}{1-\alpha} .
$$

It follows from (20) that to prove (19) it is sufficient to check inequality

$$
\frac{(1-\varepsilon(1+\Delta) \alpha)(a-1)}{1-\alpha}+\frac{2 \delta a^{\frac{1}{1-\alpha}}}{(c(1-\alpha))^{\frac{1}{1-\alpha}}}<\frac{(1+\varepsilon)(a-1)}{1-\alpha}
$$

for some $\delta>0$.

Since $|(1+\Delta) \alpha|<1$ by the assumptions of the lemma, inequality (21) is true for sufficiently small $\delta$. Lemma 3.3 is proved.

The following statement is a simple corollary of Lemma 3.3.

Lemma 3.4. Let $\varepsilon \in(0,1), a>1, \Delta \in\left(0, \frac{1+\alpha}{-\alpha}\right) \cap\left(0, \frac{1}{\varepsilon}-1\right)$ be arbitrary. Assume that (i) $\delta=\delta(\varepsilon, a, \Delta)>0$ is selected from Lemma 3.3

(ii) inequality (14) is true for some $n_{0}$,

(iii) $|g(t)| \leq \delta t^{\frac{1}{1-\alpha}}, t \geq a^{n_{0}}$.

Then

$$
\begin{gathered}
(1-\varepsilon(1+\Delta)) \leq \liminf _{t \rightarrow \infty} \frac{y(t)}{(c(1-\alpha) t)^{\frac{1}{1-\alpha}}} \leq \\
\limsup _{t \rightarrow \infty} \frac{y(t)}{(c(1-\alpha) t)^{\frac{1}{1-\alpha}}} \leq(1+\varepsilon) a^{\frac{1}{1-\alpha}} .
\end{gathered}
$$

Indeed, it follows from Lemma 3.3 that for all $n \geq n_{0}$ we have (15). So,

$$
\begin{gathered}
\limsup _{t \rightarrow \infty} \frac{y(t)}{(c(1-\alpha) t)^{\frac{1}{1-\alpha}}} \leq \limsup _{n \rightarrow \infty} \frac{\sup _{t \in\left[a^{n}, a^{n+1}\right]} y(t)}{\left(c(1-\alpha) a^{n}\right)^{\frac{1}{1-\alpha}}} \leq \\
\limsup _{n \rightarrow \infty} \frac{(1+\varepsilon)\left(c(1-\alpha) a^{n+1}\right)^{\frac{1}{1-\alpha}}}{\left(c(1-\alpha) a^{n}\right)^{\frac{1}{1-\alpha}}}=(1+\varepsilon) a^{\frac{1}{1-\alpha}} .
\end{gathered}
$$

The inequality for lim inf is proved similarly.

Theorem 3.2. Assume that $\lim _{t \rightarrow \infty} \frac{g(t)}{t^{\frac{1}{1-\alpha}}}=0$ and the solution of (9) is such that

$$
\liminf _{t \rightarrow \infty} y(t)>0 .
$$

Then

$$
\lim _{t \rightarrow \infty} \frac{y(t)}{(c(1-\alpha) t)^{\frac{1}{1-\alpha}}}=1 .
$$

Proof. Let $\varepsilon>0, \Delta \in\left(0, \frac{1+\alpha}{-\alpha}\right) \cap\left(0, \frac{1}{\varepsilon}-1\right), a>1$ be arbitrary, and $\delta=\delta(\varepsilon, a, \Delta)$ be from Lemma 3.3. Select $n$ such that

$$
\inf _{t \geq a^{n}} y(t)>0, \sup _{t \geq a^{n}} \frac{|g(t)|}{t^{\frac{1}{1-\alpha}}} \leq \frac{\delta}{2}
$$

Let

$$
x(t)=\left(c(1-\alpha) a^{n}\right)^{\frac{1}{1-\alpha}}+c \int_{a^{n}}^{t} x^{\alpha}(s) d s+g(t)-g\left(a^{n}\right) .
$$

Note that $x(t)>0, t \geq a^{n}$ by Lemma 3.3 . 
ON PERTURBATIONS OF AN ODE WITH NON-LIPSCHITZ COEFFICIENTS BY A SMALL SELF-SIMILAR NOISE10

It follows from Lemma 3.4 that

$$
(1-\varepsilon(1+\Delta)) \leq \liminf _{t \rightarrow \infty} \frac{x(t)}{(c(1-\alpha) t)^{\frac{1}{1-\alpha}}} \leq \limsup _{t \rightarrow \infty} \frac{x(t)}{(c(1-\alpha) t)^{\frac{1}{1-\alpha}}} \leq(1+\varepsilon) a^{\frac{1}{1-\alpha}} .
$$

Assume that $y\left(a^{n}\right) \geq x\left(a^{n}\right)$. Then by the comparison theorem $y(t) \geq x(t)>0, t \geq a^{n}$. Since $\alpha<0$, we have

$$
x(t)-y(t) \leq x\left(a^{n}\right)-y\left(a^{n}\right)+\int_{a^{n}}^{t}\left(x^{\alpha}(s)-y^{\alpha}(s)\right) d s \leq x\left(a^{n}\right)-y\left(a^{n}\right), t \geq a^{n} .
$$

Similarly, if $y\left(a^{n}\right) \leq x\left(a^{n}\right)$, then $x(t)-y(t) \geq x\left(a^{n}\right)-y\left(a^{n}\right), t \geq a^{n}$. Hence, in any case

$$
|x(t)-y(t)| \leq\left|x\left(a^{n}\right)-y\left(a^{n}\right)\right|, t \geq a^{n} .
$$

Therefore

$$
(1-\varepsilon(1+\Delta)) \leq \liminf _{t \rightarrow \infty} \frac{y(t)}{(c(1-\alpha) t)^{\frac{1}{1-\alpha}}} \leq \limsup _{t \rightarrow \infty} \frac{y(t)}{(c(1-\alpha) t)^{\frac{1}{1-\alpha}}} \leq(1+\varepsilon) a^{\frac{1}{1-\alpha}} .
$$

Since $\varepsilon>0, \Delta \in\left(0, \frac{1+\alpha}{-\alpha}\right) \cap\left(0, \frac{1}{\varepsilon}-1\right)$, and $a>1$ were arbitrary, the theorem is proved.

The following result follows from the course of Theorems' 3.1 and 3.2 proof.

Lemma 3.5. Let $h(t)=o\left(t^{\frac{1}{1-\alpha}}\right), t \rightarrow \infty$ be a non-negative function, and $y$ be a solution of (9), where $\alpha \in(-1,1)$. There is $R=R(\alpha, g, h)>0$ such that if $y\left(t_{0}\right) \geq R$ and $\left|g\left(t-t_{0}\right)\right| \leq h(t), t \geq$ 0 , then $\lim _{t \rightarrow \infty} y(t)=+\infty$.

\section{PRoof of THE MAIN RESUlts}

Theorem 4.1. Let $\widetilde{X}(t), t \geq 0$, be a solution to $S D E$

$$
\tilde{X}(t)=\int_{0}^{t}\left(c_{+} \mathbb{1}_{\widetilde{X}(s) \geq 0}-c_{-} \mathbb{I}_{\widetilde{X}(s)<0}\right)|\tilde{X}(s)|^{\alpha} d s+B(t), t \geq 0,
$$

where $c_{ \pm}>0, B$ is a cádlág stochastic process.

Suppose that

1) $\alpha \in(0,1)$ and there exists a function $h(t)=o\left(t^{\frac{1}{1-\alpha}}\right), t \rightarrow \infty$, and a (random) sequence $\left\{t_{n}\right\}$, $\lim _{n \rightarrow \infty} t_{n}=\infty$, such that

$$
\left|B(t)-B\left(t_{n}\right)\right| \leq h\left(t-t_{n}\right), t \geq t_{n} \text { a.s. }
$$

or

2) $\alpha \in(-1,0]$ and $B(t)=o\left(t^{\frac{1}{1-\alpha}}\right), t \rightarrow \infty$ a.s.

Then

$$
\begin{gathered}
\tilde{X}(t) \sim\left(c_{+}(1-\alpha) t\right)^{\frac{1}{1-\alpha}} \text { for a.a. } \omega \in\left\{\lim _{t \rightarrow \infty} \tilde{X}(t)=+\infty\right\}, \\
\tilde{X}(t) \sim-\left(c_{-}(1-\alpha) t\right)^{\frac{1}{1-\alpha}} \text { for a.a. } \omega \in\left\{\lim _{t \rightarrow \infty} \tilde{X}(t)=-\infty\right\} .
\end{gathered}
$$

The result follows from Theorems 3.1 and 3.2 and their natural modifications to the case $c<0$. Note that $\widetilde{X}$ has cádlág trajectories, so sets $\left\{\lim _{t \rightarrow \infty} \widetilde{X}(t)=+\infty\right\}$ and $\left\{\lim _{t \rightarrow \infty} \widetilde{X}(t)=-\infty\right\}$ are measurable. To solve the selection problem for a process $X_{\varepsilon}$ defined in (4) we need the following result. 
ON PERTURBATIONS OF AN ODE WITH NON-LIPSCHITZ COEFFICIENTS BY A SMALL SELF-SIMILAR NOISE11

Lemma 4.1. Assume that a locally bounded function $f:[0, \infty) \rightarrow \mathbb{R}$ is such that $f(t) \sim K t^{A}, t \rightarrow$ $\infty$, where $A>0$.

Set $g_{n}(t)=n^{-1} f\left(n^{1 / A} t\right)$. Then for any $T>0$ we have

$$
\lim _{n \rightarrow \infty} \sup _{s \in[0, T]}\left|g_{n}(s)-K s^{A}\right|=0
$$

Proof. Let $\varepsilon>0, \delta>0$ be arbitrary. Select $R>0$ such that $|f(s)| \leq R\left(1+s^{A}\right), s \geq 0$. Then

$$
\sup _{s \in[0, \delta]}\left|g_{n}(s)\right|=\sup _{s \in[0, \delta]}\left|\frac{f\left(n^{1 / A} s\right)}{n}\right| \leq \sup _{s \in[0, \delta]} \frac{R\left(1+\left(n^{1 / A} s\right)^{A}\right)}{n}=\frac{R}{n}+R \delta^{A} .
$$

Select $t_{0}$ such that

Then for sufficiently large $n$ :

$$
\left|\frac{f(t)}{K t^{A}}-1\right|<\varepsilon, t \geq t_{0}
$$

$$
\begin{gathered}
\sup _{s \in[\delta, T]}\left|g_{n}(s)-K s^{A}\right|=\sup _{s \in[\delta, T]}\left(K s^{A}\left|\frac{g_{n}(s)}{K s^{A}}-1\right|\right) \leq K T^{A} \sup _{s \in[\delta, T]}\left|\frac{g_{n}(s)}{K s^{A}}-1\right|= \\
K T^{A} \sup _{s \in[\delta, T]}\left|\frac{f\left(n^{1 / A} s\right)}{K\left(n^{1 / A} s\right)^{A}}-1\right| \leq K T^{A} \sup _{n^{1 / A} s \geq n^{1 / A} \delta}\left|\frac{f\left(n^{1 / A} s\right)}{K\left(n^{1 / A} s\right)^{A}}-1\right|= \\
K T^{A} \sup _{z \geq n^{1 / A} \delta}\left|\frac{f(z)}{K z^{A}}-1\right|<K T^{1 / A} \varepsilon .
\end{gathered}
$$

Thus

$$
\limsup _{n \rightarrow \infty} \sup _{s \in[0, T]}\left|g_{n}(s)-K s^{A}\right| \leq R \delta^{A}+K T^{1 / A} \varepsilon .
$$

Since $\varepsilon>0$ and $\delta>0$ are arbitrary, the lemma is proved.

Proof of Theorem 1.1 Let $X$ be a solution to (5). Theorem 4.1 yields that for a.a. $\omega \in\left\{\lim _{t \rightarrow \infty} X(t)=\right.$ $+\infty\}$ we have $X(t) \sim\left(c_{+}(1-\alpha) t\right)^{\frac{1}{1-\alpha}}, t \rightarrow \infty$.

Set $f=X, n=\varepsilon^{\frac{1}{(1-\alpha) \beta-1}}, A=\frac{1}{1-\alpha}, K=\left(c_{+}(1-\alpha)\right)^{\frac{1}{1-\alpha}}$ in Lemma 4.1. Assumption $\alpha+\beta^{-1}>$ 1 yields $(1-\alpha) \beta-1<0$.

Denote $\tilde{X}_{\varepsilon}(t):=\varepsilon^{\frac{-1}{(1-\alpha) \beta-1}} X\left(\varepsilon^{\frac{1-\alpha}{(1-\alpha) \beta-1}} t\right), t \geq 0$. So, it follows from Lemma 4.1 that for any $T>0$ and a.a. $\omega \in\left\{\lim _{t \rightarrow \infty} X(t)=+\infty\right\}$ we have the uniform convergence

$$
\lim _{\varepsilon \rightarrow 0+} \sup _{s \in[0, T]}\left|\widetilde{X}_{\varepsilon}(t)-\left(c_{+}(1-\alpha) t\right)^{\frac{1}{1-\alpha}}\right|=0 .
$$

Similarly, we have

$$
\lim _{\varepsilon \rightarrow 0+} \sup _{s \in[0, T]}\left|\widetilde{X}_{\varepsilon}(t)+\left(c_{-}(1-\alpha) t\right)^{\frac{1}{1-\alpha}}\right|=0
$$

for a.a. $\omega$ such that $\lim _{t \rightarrow \infty} X(t)=-\infty$.

Therefore, for a.a. $\omega \in\left\{\lim _{t \rightarrow \infty} X(t)=+\infty\right.$ or $\left.\lim _{t \rightarrow \infty} X(t)=-\infty\right\}$ we have the uniform convergence

$$
\begin{gathered}
\lim _{\varepsilon \rightarrow 0+} \sup _{s \in[0, T]} \mid \widetilde{X}_{\varepsilon}(t)-((1-\alpha) t)^{\frac{1}{1-\alpha}}\left(c_{+}^{\frac{1}{1-\alpha}} \mathbb{1}_{\lim _{t \rightarrow \infty} X(t)=+\infty}-\right. \\
\left.c_{-}^{\frac{1}{1-\alpha}} \mathbb{1}_{\lim _{t \rightarrow \infty} X(t)=-\infty}\right) \mid=0
\end{gathered}
$$


It follows from Lemma 3.5 that under assumptions of the Theorem

$$
\mathrm{P}\left(\left\{\lim _{t \rightarrow \infty}|X(t)|=\infty\right\} \triangle\left(\left\{\lim _{t \rightarrow \infty} X(t)=+\infty\right\} \cup\left\{\lim _{t \rightarrow \infty} X(t)=-\infty\right\}\right)\right)=0 .
$$

It follows from uniqueness of the solution and Corollary 2.1 that the distribution of $X_{\varepsilon}(t), t \geq 0$, equals the distribution of $\widetilde{X}_{\varepsilon}(t), t \geq 0$.

This and the convergence (23) yields the proof.

\section{EXAMPLES}

Lévy stable processes and a fractional Brownian motion satisfy assumptions 1) and 3) of Theorem 1.1, see Remarks 1.2 and 1.3. In this section we show that these processes also satisfy assumption 2) of the Theorem.

Example 1 (Symmetric Lévy stable noise). Let $B_{\beta}(t), t \geq 0$ be a symmetric $1 / \beta$-stable process. Then $B_{\beta}$ is self-similar process with parameter $\beta$.

It was mentioned in Remark 1.2 that there exists a unique solution to (3) if $\alpha+1 / \beta>1$. This solution is a strong Markov process [2, 9]. Condition 3) of Theorem 1.1] is also satisfied, see Remark 1.3 .

Let us verify that condition 2) of Theorem 1.1 is satisfied.

Remark 5.1. We give proofs only for $\alpha \in(0,1)$. The case $\alpha \in(-1,0]$ is simpler.

Let $\varepsilon>0$ be such that $\alpha+(\beta+\varepsilon)^{-1}>1$. Set $h(t)=1+t^{\beta+\varepsilon}$.

It follows from [11] that $\lim _{t \rightarrow \infty} B_{\beta}(t) / h(t)=0$ a.s.

It is easy to see that

$$
\gamma:=\mathrm{P}\left(\left|B_{\beta}(t)\right|>h(t), t \geq 0\right)>0
$$

To prove

$$
\lim _{t \rightarrow \infty}|\tilde{X}(t)|=\infty \text { a.s. }
$$

it suffices to show that

$$
\forall K>0 \mathrm{P}\left(\exists t_{0} \forall t \geq t_{0} \quad|\widetilde{X}(t)| \geq K\right)=1 .
$$

Let $K$ be fixed. It follows from Lemma 3.5 that there exists $M=M(K)$ such that

if $\omega$ and $t_{0}$ are such that $\left|\widetilde{X}\left(t_{0}\right)\right| \geq M$ and $\left|B_{\beta}\left(s+t_{0}\right)-B_{\beta}\left(t_{0}\right)\right|<h(s), s \geq 0$, then $|\tilde{X}(t)| \geq$ $K, t \geq t_{0}$.

Set

$\tau_{1}:=\inf \{t \geq 0:|\widetilde{X}(t)| \leq K\}, \sigma_{n}:=\inf \left\{t \in \mathbb{N} \cap\left[\tau_{n}, \infty\right):|\widetilde{X}(t)| \geq M\right\}, \tau_{n+1}:=\inf \left\{t \geq \sigma_{n}:\right.$ $|\widetilde{X}(t)| \leq K\}, n \geq 1$.

Then by the strong Markov property,

$$
X_{n}:=\left\{\begin{array}{l}
\tilde{X}\left(\tau_{n}\right), \tau_{n}<\infty \\
\infty, \tau_{n}=\infty
\end{array}\right.
$$

is a homogeneous Markov chain.

It is obvious that $\mathrm{P}\left(\sigma_{n}<\infty \mid \tau_{n}<\infty\right)=1$. Hence

$$
\sup _{|x| \leq K} \mathrm{P}_{x}\left(X_{1}=\infty \mid X_{0}=x\right) \geq \mathrm{P}\left(\left|B_{\beta}\left(s+\sigma_{1}\right)-B_{\beta}\left(\sigma_{1}\right)\right|>h(s), s \geq 0\right)=\gamma>0 .
$$


Therefore $\mathrm{P}\left(X_{n}<\infty\right) \leq(1-\gamma)^{n}$.

So

$$
\mathrm{P}\left(\exists n_{0}: X_{n_{0}}=\infty\right)=1
$$

This yields (25) and hence (24).

Thus, we have proved that all conditions of Theorems 4.1 and 1.1 are satisfied for $1 / \beta$-stable Lévy processes if $\alpha+1 / \beta>1$. So for a.a. $\omega$ we have either $\widetilde{X}(t) \sim\left(c_{+}(1-\alpha) t\right)^{\frac{1}{1-\alpha}}$ or $\widetilde{X}(t) \sim$ $-\left(c_{-}(1-\alpha) t\right)^{\frac{1}{1-\alpha}}$ as $t \rightarrow \infty$. Moreover $X_{\varepsilon}$ converges in distribution as $\varepsilon \rightarrow 0$ to

$$
((1-\alpha) t)^{\frac{1}{1-\alpha}}\left(c_{+}^{\frac{1}{1-\alpha}} \mathbb{I}_{\left\{\lim _{t \rightarrow \infty} \widetilde{X}(t)=+\infty\right\}}-c_{-}^{\frac{1}{1-\alpha}} \mathbb{I}_{\left\{\lim _{t \rightarrow \infty} \widetilde{X}(t)=-\infty\right\}}\right) .
$$

Example 2 (Fractional Brownian motion). Let $\left\{B_{H}(t)\right\}$ be a fractional Brownian motion with Hurst parameter $H \in(0,2)$. Then $B_{H}$ is the self-similar process with index $H$. Assume that $\alpha>0$ and $\alpha+H^{-1}>1$. Then, see Remarks 1.2 and 1.3 , assumptions 1) and 3) of Theorem 1.1 are satisfied.

Let us verify that condition 2) of Theorem 1.1 .

The solution of the equation is not a Markov process, so we cannot use method of the previous example.

Denote by $X_{x}(t), t \geq 0$, the solution of the SDE

$$
X_{x}(t)=x+\int_{0}^{t}\left(c_{+} \amalg_{X_{x}(s) \geq 0}-c_{-} \amalg_{X_{x}(s)<0}\right)\left|X_{x}(s)\right|^{\alpha} d s+B_{H}(t), t \geq 0 .
$$

Let $x<y$. Since the function $x \rightarrow\left(c_{+} \mathbb{I}_{x \geq 0}-c_{-} \mathbb{I}_{x(s)<0}\right)|x|^{\alpha}$ is increasing, the derivative $\frac{d\left(X_{y}(t)-X_{x}(t)\right)}{d t}$ is positive and the function $t \rightarrow X_{y}(t)-X_{x}(t)$ is increasing too.

The process $X_{x}(t), t \geq 0$, is defined up to a set of null measure. Set $\tilde{X}_{x}(t):=\lim \sup y \downarrow x X_{y}(t)$. It is easy to see that $\tilde{X}_{x}(t)$ is a modification of $X_{x}(t)$ which is measurable in $(t, x, \omega)$. Further we consider only this modification and omit tilde in the notations.

Lemma 5.1. For a.e. $\omega$ there exists a unique $x^{*}=x^{*}(\omega)$ such that

$$
\begin{aligned}
& \forall x>x^{*} \quad \lim _{t \rightarrow \infty} X_{x}(t, \omega)=+\infty, \\
& \forall x<x^{*} \quad \lim _{t \rightarrow \infty} X_{x}(t, \omega)=-\infty .
\end{aligned}
$$

Proof. Let $h$ and $\left\{t_{n}\right\}$ satisfy the assumptions of the theorem, the existence see in Remark 1.3. Select $R>0$ from Lemma 3.5 such that if

$$
y_{x}(t)=x+\int_{0}^{t}\left(c_{+} \mathbb{I}_{y_{x}(s) \geq 0}-c_{-} \mathbb{I}_{y_{x}(s)<0}\right)\left|y_{x}(s)\right|^{\alpha} d s+g(t), \quad t \geq 0,
$$

with $|x|>R$ and $|g(t)| \leq h(t), t \geq 0$, then $y_{x}(t), t \geq 0$, never changes the sign and $\lim _{t \rightarrow \infty}\left|y_{x}(t)\right|=$ $\infty$.

It follows from the monotonicity of $X_{x}(t)$ in $x$ that to prove the Lemma it is sufficient to show that for every pair $x_{1}<x_{2}$

$$
\mathrm{P}\left(\lim _{t \rightarrow \infty} X_{x_{1}}(t)=-\infty \text { or } \lim _{t \rightarrow \infty} X_{x_{2}}(t)=+\infty\right)=1 .
$$

Assume the converse. Then there exists $\omega$ such that

$$
\forall n \geq 1\left|B_{\beta}(t)-B_{\beta}\left(t_{n}\right)\right| \leq h\left(t-t_{n}\right), t \geq t_{n},
$$


and

$$
\forall n \geq 1\left|X_{x_{1}}\left(t_{n}\right)\right| \leq R,\left|X_{x_{2}}\left(t_{n}\right)\right| \leq R
$$

where $R$ is as before.

Hence for this $\omega$

$$
\exists \varepsilon_{0}>0 \forall n \geq 1 \forall t \in[0,1] \frac{d\left(X_{x_{2}}\left(t_{n}+t\right)-X_{x_{1}}\left(t_{n}+t\right)\right)}{d t} \geq \varepsilon_{0} .
$$

So

$$
\forall n \geq 1 X_{x_{2}}\left(t_{n}+1\right)-X_{x_{1}}\left(t_{n}+1\right) \geq X_{x_{2}}\left(t_{n}\right)-X_{x_{1}}\left(t_{n}\right)+\varepsilon_{0} .
$$

Without loss of generality we may assume that $t_{n+1}-t_{n} \geq 1, n \geq 1$.

Therefore

$$
X_{x_{2}}\left(t_{n}\right)-X_{x_{1}}\left(t_{n}\right) \geq x_{2}-x_{1}+n \varepsilon_{0} \rightarrow \infty, n \rightarrow \infty .
$$

This contradiction proves the Lemma.

It follows from Lemma 5.1 that there is a at most countable set $A \subset \mathbb{R}$ such that

$$
\forall x \in A \quad \mathrm{P}\left(\lim _{t \rightarrow \infty}\left|X_{x}(t)\right| \neq \infty\right)>0 .
$$

Fractional Brownian motion has stationary increments. So, if for some $x_{0} \in \mathbb{R}, t>0$

$$
\mathrm{P}\left(X_{x_{0}}(t) \notin A\right)=1
$$

then

$$
\mathrm{P}\left(\lim _{t \rightarrow \infty}\left|X_{x_{0}}(t)\right|=\infty\right)=1 .
$$

By Girsanov's theorem [3], the distribution of $X_{x_{0}}(t)$ is absolute continuous for any $x_{0} \in \mathbb{R}, t>0$. Hence (26) is satisfied for any $x_{0} \in \mathbb{R}$. So, Theorem 1.1 is applicable in the case when $\alpha>0$ and a self-similar process $B_{\beta}$ is an $\mathrm{fBm}$.

\section{REFERENCES}

[1] Engelbert, H. J. And Schmidt, W. 1991. Strong Markov Continuous Local Martingales and Solutions of One-Dimensional Stochastic Differential Equations (Part III). Mathematische Nachrichten, 151(1): 149-197.

[2] Tanaka, H., Tsuchiya, M., And Watanabe, S. 1974. Perturbation of drift-type for Lévy processes. Journal of Mathematics of Kyoto University, 14(1): 73-92.

[3] Nualart, D. And OuKnine, Y. 2002. Regularization of differential equations by fractional noise. Stochastic Processes and their Applications, 102(1): 103-116.

[4] BAFICO, R. AND BALDI, P. 1982. Small random perturbations of Peano phenomena. Stochastics, 6( 3): $279-292$.

[5] Flandoli, F. 2010. Random Perturbation of PDEs and Fluid Dynamic Models. Lecture Notes in Mathematics, 2015, Springer.

[6] Flandoli, F. 2009. Remarks on uniqueness and strong solutions to deterministic and stochastic differential equations. Metrika, 69(2-3): 101-123.

[7] Delarue, F., Flandoli, F., and Vincenzi, D. 2014. Noise prevents collapse of Vlasov-Poisson point charges, Communications on Pure and Applied Math., 67(10): 1700-1736.

[8] Buckdahn, R., Ouknine, Y., And Quincampoix, M. 2009. On limiting values of stochastic differential equations with small noise intensity tending to zero. Bull. Sci. Math. 133(3): 229-237.

[9] Portenko N.I. 1994. Some perturbations of drift-type for symmetric stable processes. Random Operators and Stochastic Equations, 2(3): 211-224, 1994.

[10] Pilipenko, A. AND Proske, F. 2015. On a selection problem for small noise perturbation in multidimensional case. arXiv preprint, arXiv:1510.00966.

[11] Khintchine, A. 1938. Zwei Sätze über stochastische Prozesse mit stabilen Verteilungen, Rec. Math. [Mat. Sbornik] N.S., 3(45):3, 577 Ü584. 
[12] Kono, N. 1983. Iterated log type strong limit theorems for self-similar processes. Proceedings of the Japan Academy, Series A, Mathematical Sciences, 59(3): 85-87.

[13] TAKASHIMA, K. 1989. Sample path properties of ergodic self-similar processes. Osaka Journal of Mathematics, 26(1): 159-189.

[14] Chen, Z. Q., WAng, L. 2016. Uniqueness of stable processes with drift. Proceedings of the American Mathematical Society, 144(6), 2661-2675.

[15] Gikhman, I.I. And Skorokhod, A.V. Stochastic differential equations. (Russian) Naukova Dumka, Kiev. 1968. - 354 p.; English translation - Ergebnisse der Mathematik und ihrer Grenzgebiete, Band 72. - SpringerVerlag. New York; Heidelberg. 1972. - viii+354 p.

[16] Keller, G., Kersting, G., And Rosler, U. 1984. On the asymptotic behaviour of solutions of stochastic differential equations, Z. Wahrsch. Verw. Geb., 68(2): 163 Ü184.

[17] Buldygin, V. V., Klesov, O. I., Steinebach, J. G., And Tymoshenko, O. A. 2008. On the $\varphi$ asymptotic behaviour of solutions of stochastic differential equations. Theory of Stochastic Processes, 14 (30), no. 1: 11 Ü29.

[18] Flandoli, F. And Hogele, M. 2014. A solution selection problem with small symmetric stable perturbations. arXiv preprint, arXiv:1407.3469

Institute of Mathematics of Ukrainian National ACAdemy of Sciences; National Technical UNIVERSITY OF UKRAINE “KPI”

DEPARTMENT OF MATHEMATICS, UNIVERSity OF OSLO 